\title{
Impact of heterogeneous network controlled handovers on multi-mode mobile device design
}

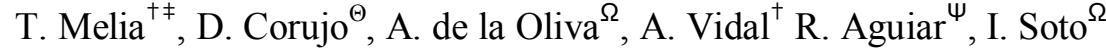 \\ ${ }^{\dagger}$ NEC Europe, Network Laboratories, Heidelberg, Germany, Email: \{melia,vidal\}@netlab.nec.de \\ ${ }^{\ddagger}$ Institute of Informatics, University of Goettingen, Goettingen, Germany \\ ${ }^{\Omega}$ Universidad Carlos III de Madrid, Madrid, Spain, Email: \{isoto,aoliva\}@it.uc3m.es \\ ${ }^{\Psi}$ Universidade de Aveiro, Aveiro, Portugal, Email: ruilaa@det.ua.pt \\ ${ }^{\Theta}$ IT Aveiro, Aveiro, Portugal, Email: dcorujo@av.it.pt
}

\begin{abstract}
The availability of multiple technologies, with micro and macro wireless cells, for network access combined with terminals capable of exploiting such diversity in wireless access requires the development of new mechanisms for optimized handover procedures. Appealing solutions should support network controlled handovers through heterogeneous technologies, preferably combined with a cross-layers two/three design. The IEEE 802.21 working group is currently standardizing the methods and the protocol potentially able to provide such a solution. In this paper we analyze the impact of signaling timing on network controlled handovers execution and performance in this environment. Through an extensive simulation study, we obtain results, that can be exploited in both terminal and handover procedure designs.
\end{abstract}

Index Terms-Cross layer design, Handover thresholds, IEEE 802.21, Heterogeneous Network Controlled Handovers

\section{INTRODUCTION}

$\mathrm{R}$ ecently there has been an increasing interest, from both mobile operators and standardization bodies, in defining advanced network support for functionalities traditionally located in mobile devices [1]. The Internet Protocol (IP), being the common convergence layer for heterogeneous networking, opens new business opportunities enabling mobile terminals equipped with several wireless/wired access technologies to maintain single/multi path Internet connectivity. In such potentially complex scenarios, the terminal might not have the possibility or capability to gather information related to neighboring cells as well as available resources of the surrounding access networks. Hence, it is desirable to relocate complexity from the terminal to the network by implementing functions for accurate network selection and optimized handover decision/execution, allowing the network to better manage resources and the users. The potential complexity of the communication between such network functionalities and the terminal requires a common interface, abstracting technology specific functionalities.

The upcoming IEEE 802.21 standard [2], currently under

This work was supported in part by IST FP6 Integrated Project DAIDALOS. DAIDALOS receives research funding from the European Community's Sixth Framework Program. Apart from this, the European Commission has no responsibility for the content of this paper. development, specifies the Media Independent Handover (MIH) layer, a function able to gather heterogeneous information and enhance the handover decision. This layer, implemented in entities as a MIH Function (MIHF), provides a cross-layer design that allows abstraction of the underlying technologies to local and remote upper layers. This abstraction allows information gathering about the current status of the different links, through link layer events, and control the link behavior, through the use of command primitives. This information can be conveyed to the network through $\mathrm{MIH}$ signaling using, for instance, a layer three (L3) approach. The specification of such a protocol is currently being defined [3].

The signaling exchange between the mobile terminal and the network-located functions of optimized network selection has an important role. Access network selection can either be implemented in the terminal or in the core network. This paper relies on the latter approach focusing on the communication between the terminal and network, as well as associated operations for handover target selection implemented in the network. The signaling itself can be continuously sent to requesting entities (i.e. the $\mathrm{MIH}$-enabled network point of decision) or can be triggered by threshold crossing, an event typically associated with transmitted and received power considerations. A typical scenario would be a media dependent link event being generated in the terminal, (e.g. because changed radio conditions require higher transmission power), collected in the local MIHF and sent to the remote MIHF located at the network decision point.

Notification of remote events is a time sensitive operation, especially in multimode terminals, where communication paths and related delays depend on the specific technology involved in the process. This paper analyses and evaluates how the handover is affected when using remote IEEE 802.21 signaling at different starting opportunities: either start the handover signaling upon cell discovery by the mobile terminal, or only when a pre-configured threshold has been crossed.

The remainder of the paper is organized as follows. Section II reviews related work. Section III describes the network technology and associated signaling for the currently relevant case of mixed 3G/WLAN networks. Section IV describes the framework design and terminal architecture. Section V and VI respectively present the simulation setup and the results 
obtained, and section VII presents a study on the effect of transmission power in the implemented model, showing how signal threshold adjustments can potentially be used for optimum network performance. Finally, concluding remarks are presented in section VIII.

\section{RELATED WORK}

Media Independent Handover frameworks have recently been subject of studies considering the direction and growth of heterogeneous environments. Generalized models [4] have been defined where abstractions about layer two (L2) triggers are provided across heterogeneous wireless interfaces. These triggers can be used in the handoff process, providing information about events that help L3 entities and above to rationalize their handover activities. Along these lines, the IEEE 802.21 allows evaluation of different links information in a joint way, collected in environments where the wide coverage of next-generation cellular systems is combined with the advantage of high bandwidth and low cost WLANs, where vertical handovers can occur [5], enabling the users to make the most of wireless IP communication.

Work has also been done on using MIH services as a way to reduce handover latency [6] as well as supporting Network Initiated Handovers (NIHO) [7]. However, no analysis on the impact of signaling timing and its inherent performance considerations is ever performed.

The contribution of this paper resides in the analysis of such timing issues when network control for heterogeneous (3G and WLAN technology) device mobility is required, focusing on the starting point of the signaling and not on its duration. The network selection procedure requires event or time based reports evaluation, in order to determine mobile terminal location and handover target selection. The paper presents the advantages, in terms of performance, of an event based approach (following the IEEE 802.21 design) and evaluates two different strategies for timely event triggering.

\section{IEEE 802.21 BASED EVENT SIGNALING}

IEEE 802.21 aims at providing the means to facilitate and improve the intelligence for handover execution. It adds a technology independent function, the MIHF, optimizing the communication between different entities, either locally (the terminal, or a network component) or remotely (between network functions and the terminal). The MIH is composed of three services: 1) the Media Independent Event Service (MIES) which provides classification, filtering and reporting of events; 2) the Media Independent Command Service (MICS) which enables local or remote high level entities to control, manage and send actions to lower layers; 3) The Media Independent Information Service (MIIS) which provides details on the characteristics and services available in the serving and surrounding networks. These three services allow collection and sharing of heterogeneous information for handover optimization, as well as the means to command those handovers, in a technology independent way. The standard also supports several mobility schemes. In our work, we opted to use Mobile IP (MIP), which, due to space limitations, the authors is background knowledge for the reader.

The IEEE 802.21 communication reference model specifies interfaces between mobile devices and points of attachment to the network and between network nodes in the network. A MIH Point of Service (PoS) is a network MIH-enabled entity that exchanges MIH signaling with a MIH-enabled terminal providing, for instance, MIIS information services located deeper in the access network. Transport definition for MIH messages is currently under discussion, but proposals supporting the use of UDP as a mean to carry MIH signaling [8] exist, which is the approach of used in this framework.

Figure 1 depicts the signaling flow considered in the paper, exchanged between the Mobile Node (MN) and the PoS supporting network initiated handovers in our case of interest, and heterogeneous network with $3 \mathrm{G}$ and WLAN technologies. The impact of this signaling in the terminal's architecture is presented in Section V.

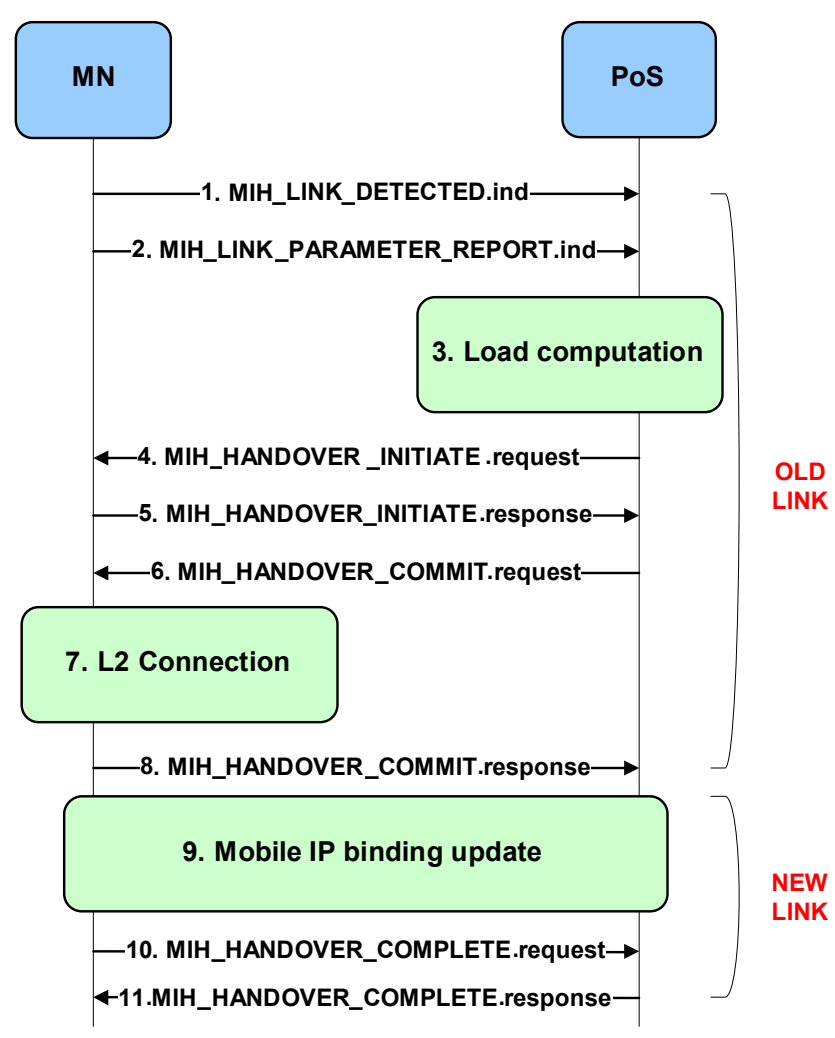

Figure 1 IEEE 802.21 signalling for Network Initiated Handover between $3 \mathrm{G}$ and WLAN including mobility related protocol (Mobile IP).

The signaling flow for the $3 \mathrm{G} \rightarrow$ WLAN handover supposes a MN connected to $3 \mathrm{G}$ and approaching a WLAN cell. As soon as an access point (AP) is detected, the MIHF at the MN receives a corresponding indication from the link layer and sends message (1) to the PoS, encoding the MAC address of the AP in a UDP packet. This message is followed by message (2), where signal strength information is supplied to the PoS.

The PoS is then able to query the handover target, or a network information server, about information for handover 
feasibility. This information aids in the handover decision, which is outside the scope of 802.21. In our model we opted to analyze the load value of the handover target, which is done in (3). If it is determined that a handover should occur, the PoS sends message (4) to the $\mathrm{MN}$ requesting for handover initiation. The MN then replies with message (5) informing if the handover is possible or not. The PoS, upon reception of this message, sends message (6) indicating commitment to carry on the handover. The $\mathrm{MN}$ processes this datagram in the MIHF, sending locally a link command to the wireless interface, in step (7). Upon successful L2 connection, message (8) is sent to the PoS. If the signal strength conditions are still favorable, the MN can execute a L3 handover (9) (a Mobile IP Registration) through the new link. Upon successful Mobile IP Registration, message (10) is sent to the PoS, which replies with message (11). Finally the MN is able to receive L3 traffic as result of the Mobile IP binding procedure.

In the case of WLAN $\rightarrow 3 \mathrm{G}$, the $\mathrm{MN}$ is associated to an $\mathrm{AP}$ and continuously evaluates the signal level supplied by beacon messages. When the WLAN $\rightarrow 3 \mathrm{G}$ threshold value is crossed, the MIH sends a Link_Parameters_Report (2) to the PoS, indicating deterioration of the received signal level. This will start a signaling exchange with the same messages and sequence as the $3 \mathrm{G} \rightarrow$ WLAN handover, except for (1) MIH_Link_Detected that is omitted, since the $3 \mathrm{G}$ leg is assumed always active (i.e. Packet Data Protocol (PDP) context always active).

\section{FrameWork Design}

Figure 1 describes the signaling exchange between the network and the terminal, for our target case of $3 \mathrm{G}$ and WLAN technologies. In the following section we focus on the terminal design (including L2 functionalities) and its integration with the L3 signaling.

\section{A. Thresholds}

The terminal's intelligence relies on several thresholds across the signal strength evaluation, as can be seen in Figure 2. While connected to the $3 \mathrm{G} \mathrm{leg}$, the terminal is able to collect probe responses and beacons from the access points, evaluating the received signal strength indication (RSSI). Two thresholds are defined, namely the association threshold and the $3 G \rightarrow W L A N$ threshold. The first one refers to the mean signal strength required for the terminal's intelligence to decide to connect to an access point. The second one refers to the mean signal strength required for the terminal to decide that a successful $3 \mathrm{G} \rightarrow$ WLAN handover is possible. Furthermore, when connected to the network through the WLAN leg, a $W L A N \rightarrow 3 G$ handover threshold is defined to determine when the signal strength conditions require a handover to the $3 \mathrm{G}$ leg.

It is worth noting in Figure 2 that the $3 G \rightarrow W L A N$ threshold is defined as being greater (in $\mathrm{dBm}$ ) than the $W L A N \rightarrow 3 G$ threshold for zero-packet loss, as analyzed in the configuration of [9] and [10]. Also, the association threshold is defined as being lower (in $\mathrm{dBm}$ ) than the $W L A N \rightarrow 3 G$ threshold.

\section{B. Operational Modes}

Depending on when (time-wise) the L3 signaling is triggered, two different operational modes have been implemented, hereinafter referred as Operational Mode A (OM.A) and Operational Mode B (OM.B). These two modes execute the signaling model presented in section III, but differ in the timing of occurrence of certain messages, more specifically in the point at which the IEEE 802.21 remote signaling related to L2 connection execution is started. In OM.A the signaling to the PoS is triggered at the configured $3 G \rightarrow W L A N$ threshold, and in OM.B the signaling to the PoS is started at a fixed value of $-80 \mathrm{dBm}$, the association threshold. Both Operational Modes have been implemented to compare the effect of how the timing of the signaling messages affects handover procedures. Figure 2 visualizes the correlation between events (WLAN cell connection) and signaling triggers to the network. It is important to note the splitting of the signaling between cell detection, and associated RSSI report, and the signaling for effective L3 handover, including Mobile IP binding update. In fact, as can be seen later, one of the metrics evaluated for the performance study is the number of L2 connections not followed by a successful L3 handover.

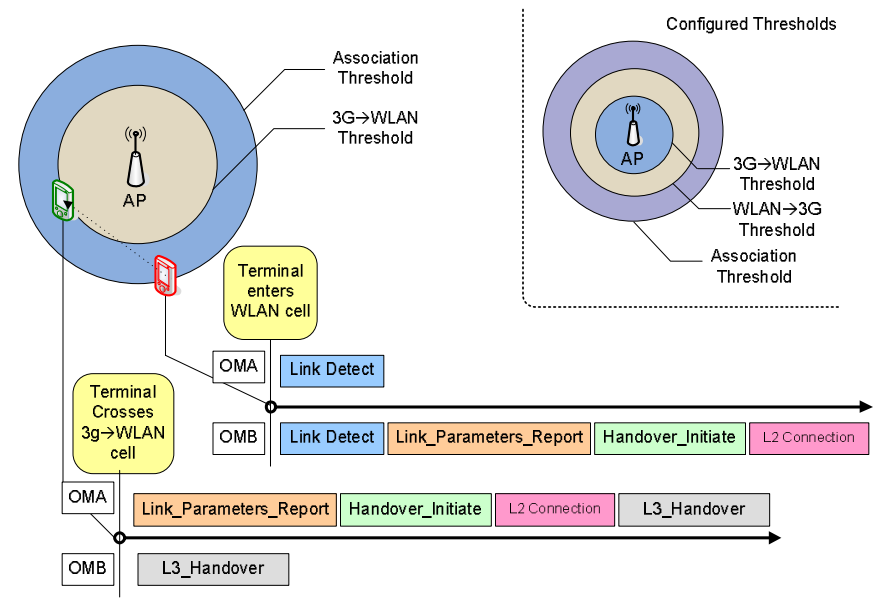

Figure 2 Different signalling stages for both operational modes

In OM.A, after cell detection, the terminal only triggers IEEE 802.21 signaling related to the L2 connection, i.e. message (2), at the $3 G \rightarrow W L A N$ threshold. So, in this mode, the terminal has to move within reach of a WLAN cell and the signal has to cross a certain configured threshold in order to execute a L2 connection, which is followed by the MIP binding update process. The nature of these operations ensures that good signal level conditions are met before the handover is executed, avoiding unnecessary handovers.

In OM.B the L2 connection related signaling is promptly sent when the association threshold is crossed, resulting in an earlier L2 connection. So, in this mode, when the signal strength crosses the $3 G \rightarrow W L A N$ threshold, the L2 connection has already been executed and the MIP binding update process can start right away. Note that the traffic flows through the old leg until the L3 handover is completed.

For both modes, in order to maintain the handover's 
feasibility, upon receiving indication from the network to commit to the handover, the terminal's intelligence executes an active scan of the wireless environment. This procedure, executed in message (7) of Figure 1, guarantees the signal strength hasn't deteriorated while waiting for the network handover command.

\section{Simulation Setup}

The simulation setup is based in the one implemented in [9] and [10]. For space limitation we report hereinafter only a brief overview of the setup. The study was conducted by simulating the movement of a $\mathrm{MN}$ attached to a $3 \mathrm{G}$ network and performing several handovers between $3 \mathrm{G}$ and localized WLAN hotspots, varying terminal speed and coverage threshold values. The movement pattern selected is the Random Waypoint Mode. The MN moves between uniformly distributed waypoints, at speeds of $2 \mathrm{~m} / \mathrm{s}, 5 \mathrm{~m} / \mathrm{s}$ and $10 \mathrm{~m} / \mathrm{s}$.

The implemented model exploits the possibilities given by the IEEE 802.21 specification in inter-technology handovers, where a MIH enabled network decision point assists the Mobile Node in handover decision, through information sharing using MIH signaling between the MN and the PoS.

The simulated scenario consists of an environment with a partial area of non-overlapping WLAN cells and full coverage of $3 \mathrm{G}$ technology. The WLAN coverage is supplied by Access Points, each connected to an Access Router. The scenario also features a Home Agent for the MIP binding update process, a video server which streams video traffic to the $\mathrm{MN}$, and the PoS which is the network entity that exchanges MIH messages with the MN.

The OMNeT $++^{1}$ simulator has been used as the primary tool for this study, with each simulation run for 60 random seeds. The WLAN Model used is the default in OMNeT++, based on free space losses with shadowing and a variable exponential coefficient. Each simulation was run with $3 G \rightarrow W L A N$ and $W L A N \rightarrow 3 G$ thresholds varying between $75 \mathrm{dBm}$ and $-65 \mathrm{dBm}$. The $3 \mathrm{G}$ channel is modelled as a Point to Point Protocol (PPP) channel, as in [9] and [10], sharing the same characteristics and assuming that the PDP context is always active.

As the scope of our work is the analysis of the impact of signalling (the framework considers the upcoming IEEE 802.21) for network controlled mobility and its integration with L2 technology we propose the following metrics:

- Mean percentage of L2 connections without MIP registration.

- Mean number of 3G $\rightarrow$ WLAN handovers.

- Mean number of WLAN $\rightarrow 3 \mathrm{G}$ handovers.

- Mean wireless utilization time.

The first metric indicates the percentage of handovers where a L2 connection was executed but the L3 handover was non-existing, due to signal deterioration or terminal's movement out of the wireless cell. The selected operational mode has a significant impact on this metric. The second and

\footnotetext{
${ }^{1}$ http://www.omnetpp.org
}

third metrics indicate the number of $3 \mathrm{G} \rightarrow$ WLAN and WLAN $\rightarrow 3 \mathrm{G}$ handovers per simulation run. Due to scenario design the two metrics are symmetric (i.e. a $3 \mathrm{G} \rightarrow$ WLAN handover is always followed by a WLAN $\rightarrow 3 \mathrm{G}$ handover). The last metric refers to the time the terminal was connected to the WLAN leg, per handover. Packet loss is not considered, since zero-packet loss threshold configuration (taken from [9]) is always used.

\section{Results Evaluation}

In this section we compare OM.A and OM.B against the above mentioned metrics.

Figure 3 represents the percentage of L2 connections not followed by a L3 Mobile IP registration (thus failed handovers), for both operational modes. The $3 G \rightarrow W L A N$ threshold variation from -75 up to $-65 \mathrm{dBm}$ shows us that, for $10 \mathrm{~m} / \mathrm{s}$, the percentage of L2 associations not followed by a successful L3 handover increases up to almost $80 \%$ and $60 \%$, for OM.A and OM.B respectively. The curves follow a similar increasing behavior for 2 and $5 \mathrm{~m} / \mathrm{s}$, although not so accentuated. As can be noted, the curves show a trend to increase while the $3 G \rightarrow W L A N$ threshold value is increased.

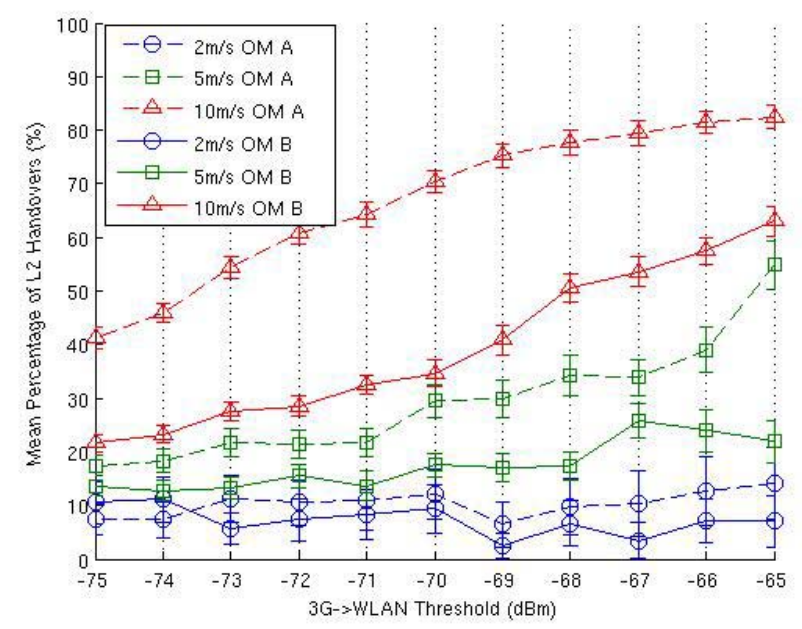

Figure 3 Mean percentage of L2 connections not followed by a L3 handover when WLAN $\rightarrow 3 \mathrm{G}$ thresholds configured at $-75 \mathrm{dBm}$

This is a direct consequence of the implemented signaling. As the $3 G \rightarrow W L A N$ threshold is increased (in $\mathrm{dBm}$ ) the MN needs to be nearer to the AP to connect to the WLAN. Since the signaling handshakes that must occur are also impacted by the Round Trip Time (RTT) of the links, this interchange of information increases the probability of moving out of the cell prior to the reception of the MIH_Handover_Commit.request command. Depending on the speed of the terminal, this behavior can be dominant, as in the $10 \mathrm{~m} / \mathrm{s}$ case. The different results obtained for the two operational modes shows us that starting the 802.21 handover related signaling as soon as a WLAN cell is detected (i.e. OM.B) decreases the number of failed L3 handovers in $20 \%$, for $10 \mathrm{~m} / \mathrm{s}$. This decrease is also verified for the $2 \mathrm{~m} / \mathrm{s}$ and $5 \mathrm{~m} / \mathrm{s}$ speeds, although not as accentuated. This behavior is particularly evident at lower threshold configurations, where the percentage of failures is 
almost reduced by half.

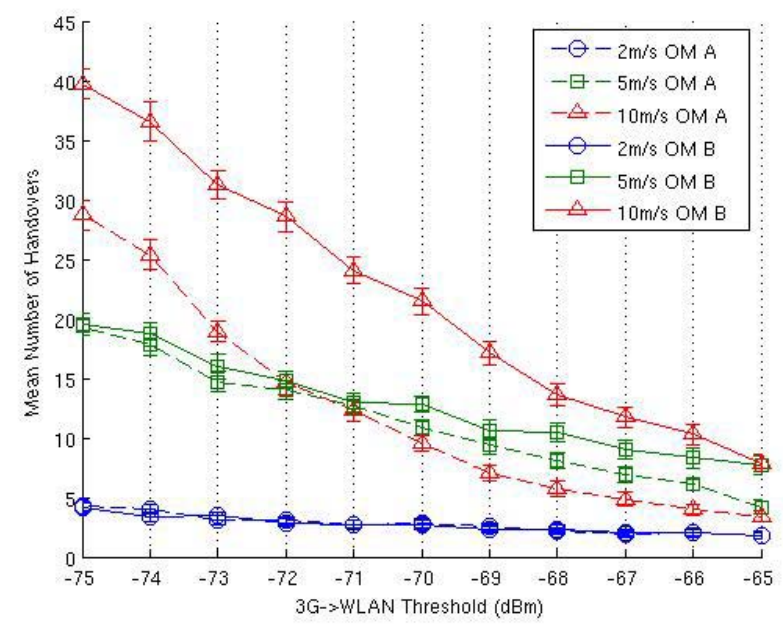

Figure 4 Mean number of $3 \mathrm{G} \rightarrow$ WLAN handovers when the WLAN $\rightarrow 3 \mathrm{G}$ threshold is configured at $-75 \mathrm{dBm}$

Figure 4 depicts the mean number of L3 handovers obtained by varying the $3 G \rightarrow W L A N$ threshold. The impact of the speed affects the metric in different ways depending on the considered configuration. At the value $-75 \mathrm{dBm}$ the number of handovers is quite large especially considering high mobility level, while decreases and converges for greater values of the threshold. The decay in the slope of the different speeds is related with the failures of performing the L3 handover shown in the previous metric. The graph shows how the values tend to converge, when the $3 G \rightarrow W L A N$ threshold is increased.

It is interesting to note that the closer the mobile node needs to be to the access point for handover execution, the lower the chance of having complete handovers. This is complementary to the previous graph in the sense that even starting the signaling at $-80 \mathrm{dBm}$, thresholds close to the -65 value affect the feasibility of the handover procedure.

Comparing both operational modes, it is visible that the number of handovers in OM.B is greater than in OM.A, especially at higher speeds. It is also visible that at higher speeds the number of handovers decreases greatly, where the $10 \mathrm{~m} / \mathrm{s}$ curve becomes lower than the respective $5 \mathrm{~m} / \mathrm{s}$ curve, for OM.A. This shows how terminal speed impacts network response time making handovers not possible. In OM.B the $10 \mathrm{~m} / \mathrm{s}$ curve always resides above the $5 \mathrm{~m} / \mathrm{s}$ curve indicating that, by executing the signaling upon cell detection, there is a greater chance that the terminal is still inside the cell when the answer arrives from the network. Simulation results confirm therefore that, for a better and cleaner protocol design, splitting of the signaling for L2 and L3 events is required, where the L2 connection is performed at the association threshold and the L3 handover at the $3 G \rightarrow W L A N$ threshold. As a final remark on the tendency of the $5 \mathrm{~m} / \mathrm{s}$ slope and $10 \mathrm{~m} / \mathrm{s}$ slope we see how OM B outperforms OM A (when crossing the $-72 \mathrm{dBm} 3 \mathrm{G}->$ WLAN threshold), reducing therefore the number of handover opportunities.

TABLE I shows the mean wireless utilization time for the different speeds, according to both operational modes.
Variations on the $3 G \rightarrow W L A N$ threshold showed a flat behavior for all speeds, and the average value is represented here. As we can see, although OM.B has a slightly higher wireless utilization time on all three speeds, the point of start for the IEEE 802.21 handover related signaling has no significant impact on this metric.

TABLE I WIRELESS UTILIZATION TIME PER HANDOVER

\begin{tabular}{|c|c|c|}
\cline { 2 - 3 } \multicolumn{1}{c|}{} & \multicolumn{2}{c|}{ Operational Mode } \\
\hline Speed & $\boldsymbol{O M} . \boldsymbol{A}$ & $\boldsymbol{O M} . \boldsymbol{B}$ \\
\hline $2 \mathrm{~m} / \mathrm{s}$ & $32.25 \mathrm{~s}$ & $32.35 \mathrm{~s}$ \\
$5 \mathrm{~m} / \mathrm{s}$ & $9.11 \mathrm{~s}$ & $9.65 \mathrm{~s}$ \\
$10 \mathrm{~m} / \mathrm{s}$ & $4.33 \mathrm{~s}$ & $4.53 \mathrm{~s}$ \\
\hline
\end{tabular}

The fact that within the same speed, the results for both operational modes are of the same order of magnitude also confirms that, when the handover is executed, performance of the configured system is maintained.

\section{ACCESS PoINT TRANSMISSION POWER IMPACT}

When considering threshold configuration and time sensitive operations, it would be desirable to implement a model able to adapt to different environments such as operator dependent network deployments (e.g. network planning). In this section we analyze the impact of transmission power on the threshold based model. A reference WLAN coverage area was chosen from the previous simulations, and new threshold values where calculated with new transmission values, maintaining the same WLAN coverage area for event triggering. Our goal is to verify the model adaptation to transmission power changes, and to analyze handover behavior differences while maintaining the same signaling triggering points. Transmission power values were taken from commercial products data sheets, complying with UMA [11].

From Figures 5-7 we can derive that the previous metrics present similar values, within their respective confidence intervals, when comparing different transmission powers in the same model and at the same speed. Figure 5 shows how the first metric, incomplete handovers, maintains a relative linear behavior in which is visible the differentiation between OM.A and OM.B, particularly at higher speeds, as noted in section VI. The same linear behavior can be noticed in Figure 6 , for the number of handovers. Figure 7, for the wireless utilization time, shows us that there is no different behavior in handover related issues caused by transmission power or speed change.

These results lead us to the conclusion that changing the APs transmission power and the threshold value configuration, maintaining the wireless coverage area, produces no changes in the results obtained. 


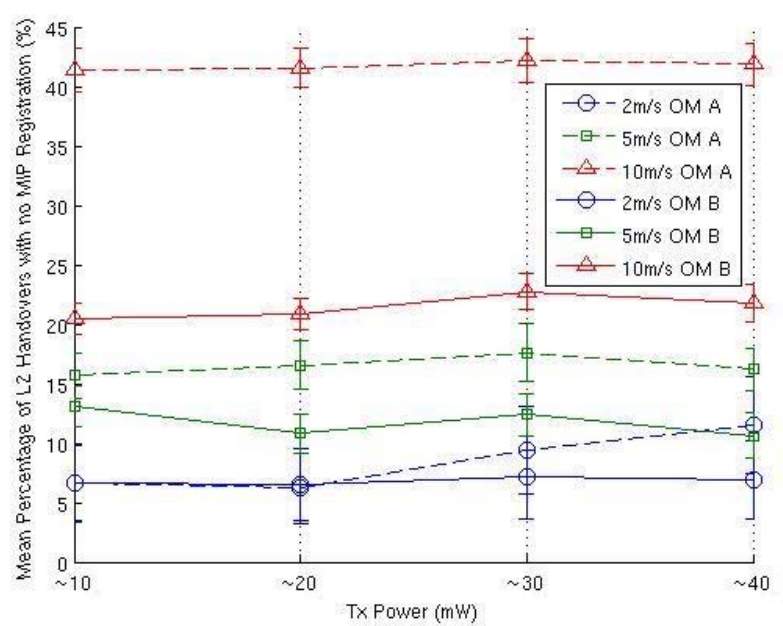

Figure 5 Mean percentage of L2 connections not followed by a L3 handover with different $\mathrm{Tx}$ and threshold values

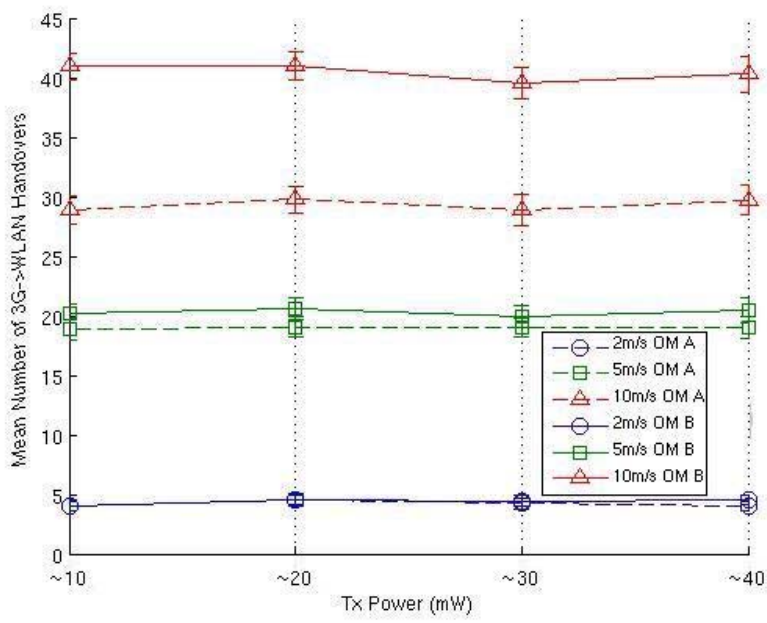

Figure 6 Mean number of $3 \mathrm{G} \rightarrow$ WLAN handovers with different Tx and threshold values

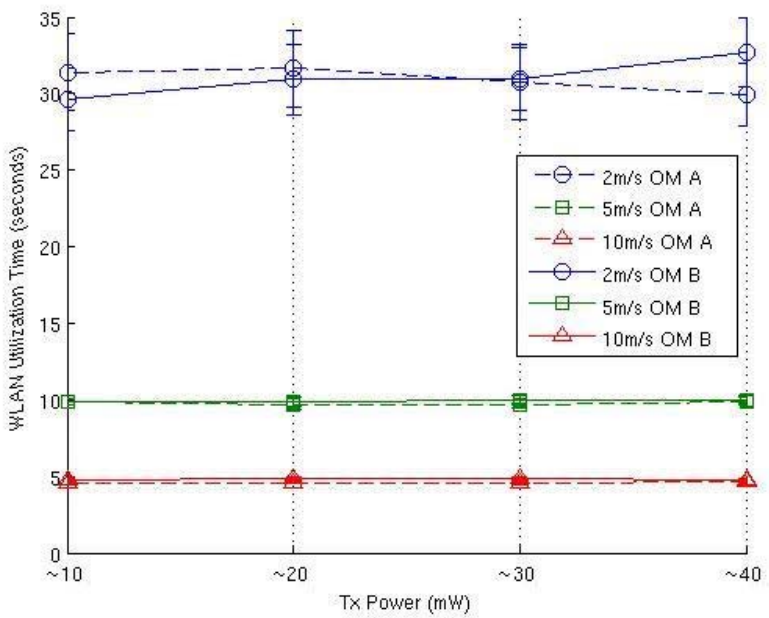

Figure 7 Wireless utilization time with different $\mathrm{Tx}$ and threshold values

Having this data in consideration, we can argue that the only factor that contributes for result differentiation is the amount of time the terminal logically resides in the WLAN cell, which is affected by threshold configuration. It is desirable that this configuration can be dynamically adapted in the $\mathrm{MN}$, through information received from the AP concerning its transmission value: this may allow an operator to dynamically adjust the coverage area of WLAN hotspots according to the density of users, but still retaining a uniform behavior in terms of handovers. This will allow operators to better configure their networks and terminals to improve handover procedures.

\section{CONCLUSION}

The paper presented the study of an optimized terminal design for heterogeneous network-controlled IP based handovers encompassing L2 and L3 integration. Through an extensive simulation study the interaction between the L3 protocol signaling and terminal's MIHF is evaluated. Two different operational modes are introduced aiming at showing challenges and different configuration possibilities. The results show the importance of splitting L2 related events reports from L3 handover procedures. To prove the general validity of our approach we also evaluated the impact of different transmitting power at the access points. Upon proper configuration of the threshold we demonstrated that the only factor impacting the handover procedure is the time the terminal resides within the WLAN cell. This important result allows mobile operators to optimally plan and deploy wireless networks while mobile devices can dynamically adapt handover thresholds to maintain optimal handover performance.

\section{REFERENCES}

[1] J. Kempf, "Goals for Network-based Localized Mobility Management (NETLMM)", Internet Draft, August 2006

[2] Draft IEEE Standard for Local and Metropolitan Area Networks: Media Independent Handovers Services (Draft 01.00). IEEE, March 2006

[3] E. Hepworth, S. Sreemanthula and S. Facciny. Ohba, "Media Independent Handovers: Problem Statement", Internet Draft, June 2006

[4] Gupta, V. and D. Johnston, "A Generalized Model for Link Layer Triggers", submission to IEEE 802.21, March 2004

[5] Q. Zhang, C. Guo, Z. Guo and W. Zhu, "Efficient mobility management for vertical handoff between WWAN and WLAN", IEEE Communications Magazine, vol. 41, Issue 11, p102-108, Nov. 2003

[6] Yoon Young An et al., "Reduction of Handover Latency Using MIH Services in MIPv6", Advanced Information Networking and Applications(AINA) 2006, vol.2 18-20, p229-234, April 2006

[7] Ok Sik Yang et al., "A Handover Framework for Seamless Service Support between Wired and Wireless Networks", Advanced Communication Technology (ICACT) 2006, vol. 3 20-22, p1791-1796.

[8] A. Rahman, U. Olvera-Hernandez, and M. Watfa. Transport of Media Independent Handover Messages Over IP. Internet Draft, June 2006

[9] T. Meila et al, "Analysis of the effect of mobile terminal speed on WLAN/3G vertical handovers", to appear in Globecom 2006, San Francisco, USA

[10] A. de la Oliva et al, "A case study: IEEE 802.21 enabled mobile terminals for optimized WLAN/3G handovers", to appear in ACM SIGMOBILE Mobile Computing and Communications Review (MC2R), 2006.

[11] "Universal Mobile Access (UMA) User Perspective (Stage 1) R 1.0.0." Alcatel, AT\&T Wireless Services, BT PLC, Cingular Wireless LLC, Ericsson AB, Kineto Wireless Inc, Motorola, Nokia, Nortel Networks, O2, Rogers Wireless, Siemens AG, Sony Ericsson, T-Mobile USA 